\title{
The brain, another potential target organ, needs early protection from SARS-CoV-2 neuroinvasion
}

\author{
Zhengqian $\mathrm{Li}^{1}$, Yuguang Huang ${ }^{2} \&$ Xiangyang Guo ${ }^{1 *}$ \\ ${ }^{1}$ Department of Anesthesiology, Peking University Third Hospital, Beijing 100191, China; \\ ${ }^{2}$ Department of Anesthesiology, Peking Union Medical College Hospital, Beijing 100730, China \\ Received March 12, 2020; accepted March 29, 2020; published online March 31, 2020
}

\begin{tabular}{ll}
\hline Citation: & Li, Z., Huang, Y., and Guo, X. (2020). The brain, another potential target organ, needs early protection from SARS-CoV-2 neuroinvasion. Sci China \\
& Life Sci 63, 771-773. https://doi.org/10.1007/s11427-020-1690-y
\end{tabular}

With the outbreak and rapid spread of the novel coronavirus disease (COVID-19) around the world, humans are engaged in a life-and-death battle with the severe acute respiratory syndrome coronavirus 2 (SARS-CoV-2), formerly known as 2019-Novel Coronavirus (2019-nCoV). Based on the existing evidence and lessons from SARS outbreak in 2003, our attention should not be confined to the general organs whose dysfunctions were relatively easy to be observed or examined such as lung, kidney, and liver; at the same time, the brain should not be neglected due to the potential neuroinvasion of SARS-CoV-2, which prompts us to keep an alert on the onset of neurological symptoms, early diagnostics, and neuroprotection.

On March 3, the National Health Commission of China issued an updated version of the diagnosis and treatment guidelines for COVID-19 (China NHCotPsRo, 2020). In the latest version of the guidelines, the pathological description was based on a small number of human histopathological samples. These showed that multiple organs are involved, including lungs, spleen and hilar lymph nodes, heart and blood vessels, liver and gallbladder, kidney, brain, adrenal gland, esophagus, stomach, and intestines. Specifically, edema and partial neuronal degeneration were observed in brain tissues (China NHCotPsRo, 2020). A proportion of COVID-19 patients in Wuhan city presented with neurologic signs such as headache (about $8 \%$ ), nausea and vomiting

*Corresponding author (email: puthmzk@hsc.pku.edu.cn)
(1\%) (Chen et al., 2020). A retrospective case series of 214 COVID-19 patients reported that up to $36.4 \%$ of patients had neurologic symptoms manifested as acute cerebrovascular diseases, consciousness impairment and skeletal muscle symptoms (Mao et al., 2020). Furthermore, a COVID-19 patient was diagnosed with viral encephalitis in Beijing Ditan Hospital by Jingyuan Liu. The presence of SARS-CoV-2 in cerebrospinal fluid was confirmed later by gene sequencing (unpublished data). As shown in Figure 1, these findings suggest that COVID-19 patients should be treated promptly to reduce complications beyond the lungs. This may decrease the mortality rate.

As a member of the family of coronaviruses (CoVs), the pathogen SARS-CoV-2 shares a highly homologous sequence with the SARS-CoV and Middle East respiratory syndrome CoV (MERS-CoV). Previous studies have shown that the brain was a major target organ for infections from almost all the betacoronaviruses, including SARS-CoV and MERS-CoV, both in patients and experimental animals (Li et al., 2020). In light of the structural similarity between SARS$\mathrm{CoV} 2$ and betacoronaviruses, it is highly suspected that SARS-CoV-2 also possesses similar neuroinvasive and neurotrophic properties. In addition, SARS-CoV-2 and SARS-CoV shared the same host receptor with the human angiotensin-converting enzyme 2 (ACE2) (Hamming et al., 2004), an important component of the brain renin-angiotensin system (RAS). The existence of an independent intrinsic cerebral RAS with all its components has been well 


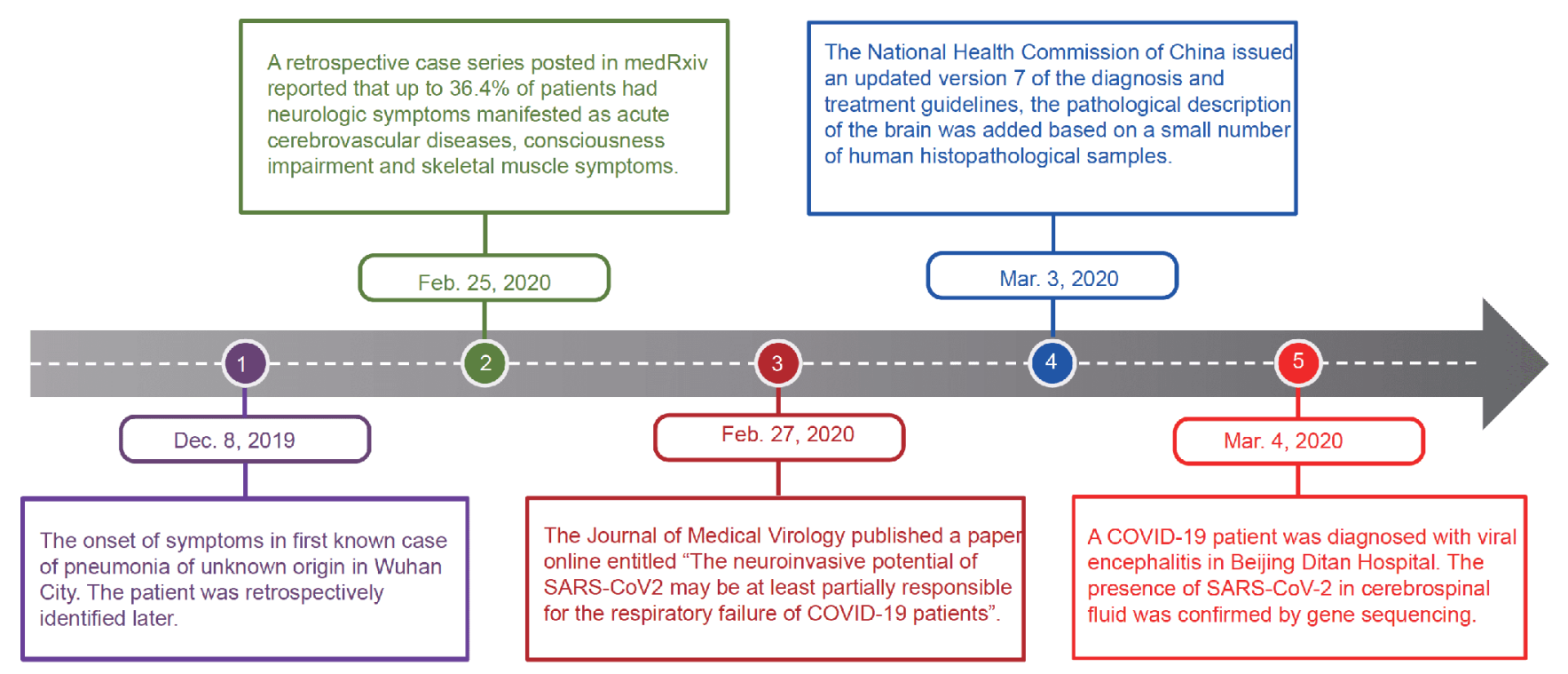

Figure 1 (Color online) A timeline of events for the notice of neurological manifestations in COVID-19 patients.

established. So far, no direct evidence of entry of SARSCoV-2 into the CNS has been reported in any international peer-reviewed journal, although some researchers have proposed that the neuroinvasive potential of SARS-CoV2 may be at least partially responsible for the respiratory failure of COVID-19 patients (Li et al., 2020). Actually, human respiratory viruses may enter the CNS through different routes involving the vasculature, the olfactory and trigeminal nerves, the cerebrospinal fluid, and the lymphatic system (Desforges et al., 2020). We suggest that the infection mechanisms previously found for SARS-CoV may be applicable for SARS-CoV-2 and we have to be vigilant about the neuroinvasion of SARS-CoV-2 in the CNS until solid pathological evidence is available.

It should be emphasized that COVID-19 patients will be facing both neuropsychological and neurological challenges. On the one hand, with the rapid transmission of life-threatening pneumonia, self-quarantine and medical observations not only increase the possibility of psychological and mental problems, including anxiety and depression, but also reduce the availability of timely psychological intervention. Even one year after the outbreak, SARS survivors still had elevated stress levels and worrying levels of psychological distress (Lee et al., 2007). On the other hand, viral dissemination in brain tissue may be accompanied by neuroinflammation and vascular endothelium dysfunction, which have been reported to contribute to cognitive impairment, especially in frail or elderly patients (Toth et al., 2017). These changes may be much worse under the hypoxic conditions of acute respiratory distress syndrome caused by SARS-CoV-2.

Awareness of the potential neuroinvasion of SARS-CoV-2 will have critical significance for the prevention and treatment of COVID-19. Early antiviral therapy with neuroprotective effects should be taken as soon as possible. For example, clear lung detoxification soup, a classical prescription of traditional Chinese medicine combination, has been shown to be an effective treatment based on syndrome differentiation (China NHCotPsRo, 2020). In addition, clinical physical examination including the pupillary light reflex and a variety of tendon reflexes, and pathogenic detection of cerebrospinal fluid should be considered, if clinically available, for timely identification and management of neurological complications. Furthermore, prompt endotracheal intubation and mechanical respiratory support at the earliest sign of ineffective noninvasive ventilation should be proposed, since the central respiratory failure progresses rapidly after the SARS-CoV-2's entry into the CNS and damages the brainstem where the pneumotaxic center is located (Li et al., 2020). It is also worth mentioning that the long-term psychological and neurocognitive implications of COVID-19 should not be ignored. Last but not the least, the effective vaccine with safety profile and antiviral drugs that can cross the blood-brain barrier are urgently needed.

Compliance and ethics The author(s) declare that they have no conflict of interests.

Acknowledgements This work was supported by Peking University "Clinical Medicine plus X" Youth Project (PKU2020LCXQ016) and the National Natural Science Foundation of China (81971012, 81873726).

\section{References}

Chen, N., Zhou, M., Dong, X., Qu, J., Gong, F., Han, Y., Qiu, Y., Wang, J., 
Liu, Y., Wei, Y., et al. (2020). Epidemiological and clinical characteristics of 99 cases of 2019 novel coronavirus pneumonia in Wuhan, China: a descriptive study. Lancet 395, 507-513.

China NHCotPsRo. (2020). The guidelines for the diagnosis and treatment of novel coronavirus (2019-nCoV) infection (trial version 7) (in Chinese). http://www.nhc.gov.cn/yzygj/s7653p/202003/46c9294a7dfe4cef80dc7f5912eb1989/files/ce3e6945832a438eaae415350a8ce964.pdf.

Desforges, M., Le Coupanec, A., Dubeau, P., Bourgouin, A., Lajoie, L., Dubé, M., and Talbot, P.J. (2020). Human coronaviruses and other respiratory viruses: underestimated opportunistic pathogens of the central nervous system? Viruses 12, 14.

Hamming, I., Timens, W., Bulthuis, M.L.C., Lely, A.T., Navis, G.J., and van Goor, H. (2004). Tissue distribution of ACE2 protein, the functional receptor for SARS coronavirus. A first step in understanding SARS pathogenesis. J Pathol 203, 631-637.

Mao, L., Wang, M., Chen, S., He, Q., Chang, J., Hong, C., Zhou, Y., Wang,
D., Li, Y., Jin, H., et al. (2020). Neurological manifestations of hospitalized patients with COVID-19 in Wuhan, China: a retrospective case series study. MedRxiv, doi: https://doi.org/10.1101/2020.02.22. 20026500.

Lee, A.M., Wong, J.G.W.S., McAlonan, G.M., Cheung, V., Cheung, C., Sham, P.C., Chu, C.M., Wong, P.C., Tsang, K.W.T., and Chua, S.E. (2007). Stress and psychological distress among SARS survivors 1 year after the outbreak. Can J Psychiatry 52, 233-240.

Li, Y.C., Bai, W.Z., and Hashikawa, T. (2020). The neuroinvasive potential of SARS-CoV2 may be at least partially responsible for the respiratory failure of COVID-19 patients. J Med Virol, in press.

Toth, P., Tarantini, S., Csiszar, A., and Ungvari, Z. (2017). Functional vascular contributions to cognitive impairment and dementia: mechanisms and consequences of cerebral autoregulatory dysfunction, endothelial impairment, and neurovascular uncoupling in aging. Am J Physiol-Heart Circulatory Physiol 312, H1-H20. 